\begin{tabular}{|c|l|}
\hline Title & Electrochemical Potential Control of Stretched Length of A u Nano-wire in Solution \\
\hline Author(s) & Kiguchi, Manabu; Konishi, Tatsuya; Murakoshi, Kei \\
\hline Citation & $\begin{array}{l}\text { Chemistry Letters, 34(10), 1336-1337 } \\
\text { https://doi.org/10.1246/1.2005.1336 }\end{array}$ \\
\hline Issue Date & 2005 \\
\hline Doc URL & http://hdl.handle.net/2115/10091 \\
\hline Type & article (author version) \\
\hline File Information & ChemLett_v34p1336.pdf \\
\hline
\end{tabular}

Instructions for use 


\title{
Electrochemical Potential Control of Stretched Length of Au Nano-wire in Solution
}

\author{
Manabu Kiguchi, Tatsuya Konishi, and Kei Murakoshi \\ Department of Chemistry, Graduate School of Science, Hokkaido Univ., Kita 10 Nishi 8, Sapporo, Hokkaido, 060-0008
}

(Received August 5, 2005; CL-051015)

Gold nano wire was mechanically fabricated in $0.1 \mathrm{M}$ $\mathrm{Na}_{2} \mathrm{SO}_{4}$ solution under electrochemical potential control. Stretched length of the gold nano-wire was evaluated from the length of the last conductance plateau showing a unit conductance quantum, $1 G_{0}=2 e^{2} / h$. Estimated wire length changed reversibly with the electrochemical potential. We have shown that the stability of the gold atomic wire could be controlled by the electrochemical potential.

Gold nano-constrictions and wires can be fabricated by mechanically controllable break junctions (MCBJ) or scanning tunneling microscopy (STM). ${ }^{1}$ When the metal nano-contact becomes to be small in an atomic scale, the conductance can be described with Landauer's formula $G=2 e^{2} / h \sum T_{i}$, where $T_{i}$ is the transmission probability of the $i$-th conductance channel and $G_{0}=2 e^{2} / h$ is the unit of quantum conductance. In the case of gold, the conduction channel is composed of single gold $6 \mathrm{~s}$ channel with the transmission of unity. As the consequence, the conductance of gold atomic wire is quantized in units of $G_{0}{ }^{1,2}$ It has been shown that quantized conductance measurements provide information on the structure of the nano-wire, such as diameter and length of the wire. ${ }^{1,2}$

Several measurements of high resolution transmission electron microscopy (TEM) proved the formation of the gold mono atomic wire at the state showing a unit conductance quantum, $1 \mathrm{G}_{0}{ }^{3}$. Detailed conductance measurements also supported the formation of one-dimensional atomic wire from the observations of characteristics conductance fluctuation. ${ }^{4}$ Most of the evaluations on the stability of the gold mono atomic wire, however, carried out in ultra high vacuum (UHV) at low temperature, ${ }^{1,4}$ in which fluctuation of environment was rather small. Possibility on the fabrication and the control in structure of the mono atomic wire at room temperature should be investigated critically for technological application on electronic devices (i.e. molecule sensor) in future.

Recently, it has been demonstrated that electrochemical method is powerful approach to fabricate metal nano-contact showing the conductance quantization. ${ }^{5-7}$ Electrochemical potential determines the potential energy of electrons of the nano-constriction, resulting in the control of the bonding strength between the metal atoms, and the interaction of the metals with molecules of surrounding medium. Shu et al. succeeded in the observation of the conductance quantization of gold nano-constriction in $0.1 \mathrm{M} \mathrm{NaClO}_{4}{ }^{5}$ We also proved the experimental evidence showing the formation of the gold mono atomic in $0.1 \mathrm{M} \mathrm{Na}_{2} \mathrm{SO}_{4}{ }^{6}$. At the $1 G_{0}$ plateau in the conductance trace, the value of the conductance showed sudden decreases and recoveries by $0.1 G_{0}$. This conductance fluctuation was attributed to the change in the number of atoms of the mono atomic wire. ${ }^{4}$ While metal nano-constrictions in electrochemical system have attracted wide attention, there is little study on the effect of the electrochemical potential on their structures and stabilities of the metal nano-wire.

The experiments were performed in a four-electrodes electrochemical cell mounted in a chamber that was filled with high purity $\mathrm{N}_{2}$ gas to reduce the effect of oxygen. The gold nano-constrictions were created by driving a STM tip into and out of contact with a gold substrate at a typical rate of $50 \mathrm{~nm} / \mathrm{s}$ in the electrochemical cell. The tip was made of a gold (diameter $\sim 0.25 \mathrm{~mm}$ ) wire coated with wax to eliminate ionic conduction. The gold substrate was $\mathrm{Au}(111)$ prepared by the flame annealing and quenching method. The electrochemical potential of the gold substrate and tip was controlled using potentiostat (Pico-Stat, Molecular Imaging Co.) with respect to the Ag/AgCl reference electrode. A $0.50 \mathrm{~mm}$ diameter Pt wire was used as a counter electrode. The electrolyte was $0.1 \mathrm{M}$ $\mathrm{Na}_{2} \mathrm{SO}_{4}$. Conductance of the gold nano-constriction was calculated based on the observed current between the tip and substrate at the potential difference of $20 \mathrm{mV}$.

Figure 1 shows typical conductance trace of the gold nano-constriction in $0.1 \mathrm{M} \mathrm{Na}_{2} \mathrm{SO}_{4}$ solution at electrochemical potential of $+0.5 \mathrm{~V}$. Conductance decreased in a stepwise fashion, and showed the last plateau at $1 G_{0}$. The length of this last plateau was as long as $0.5 \mathrm{~nm}$. Since the $1 G_{0}$ plateau corresponds to the formation of a contact with a single atom at the narrowest cross section, ${ }^{1,2}$ observed length of the plateau suggests a $0.5 \mathrm{~nm}$ long mono atomic gold wire was fabricated in solution at room temperature. The formation of mono atomic wire was supported by the conductance fluctuation observed at the $1 G_{0}$ plateau. ${ }^{4}$ Sudden decreases and recoveries (arrow in Figure 1 ) in the conductance value by $0.1 G_{0}$ at the $1 G_{0}$ plateau are the characteristic of the mono-atomic wire. ${ }^{4,6}$ Observed conductance fluctuation strongly supports the formation of gold mono atomic wire in solution. Figure 2 shows the conductance histograms of gold nano-constrictions in $0.1 \mathrm{M}$ $\mathrm{Na}_{2} \mathrm{SO}_{4}$ observed at three different electrochemical potentials. Each conductance histogram was obtained from a large number (over 3000) of individual conductance traces. In these

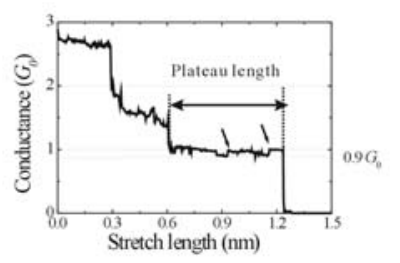

Figure 1. Typical conductance trace of gold mono atomic chain at electrochemical potential of $+0.5 \mathrm{~V}$.

conductance histograms, well-defined peaks appear at near integer multiple of $G_{0}$. At electrochemical potential of $+0.5 \mathrm{~V}$, 


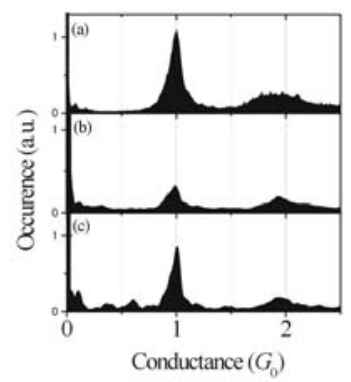

Figure 2. Conductance histograms of Au nano-constructions in $\mathrm{Na}_{2} \mathrm{SO}_{4}$ at (a) $+0.5 \mathrm{~V}$, (b) $-0.4 \mathrm{~V}$ and (c) $-0.8 \mathrm{~V}$.

well-defined peaks appeared clearly at near integer multiple of $G_{0}$. As the potential was changed from $+0.5 \mathrm{~V}$ to $-0.4 \mathrm{~V}$, the intensity of the integer peaks decreased. At more negative electrochemical potential $-0.8 \mathrm{~V}$, the intensity of the integer peaks recovered. Since the evolution of the peak at $1 G_{0}$ corresponds to the formation of mono atomic contact, thus peak intensity in the histogram reflects the stability of the gold mono atomic wire in solution. Observed electrochemical potential dependence of the histogram suggests that the stability of the gold mono atomic wire changes with the electrochemical potential.

Peak height at $1 G_{0}$ should reflect the stretched length of the gold mono atomic wire. To prove this hypothesis, we analyzed the conductance traces in terms of the length of the plateau showing $1 G_{0}$. Figure 3 shows the averaged lengths of the mono atomic wires, which were determined by the length of the plateau at $1 G_{0}$ as a function of electrochemical potential. ${ }^{1}$ The average length was shortest at $-0.4 \mathrm{~V}$. Potential at more negative or more positive than $-0.4 \mathrm{~V}$, the length became longer. It was confirmed that this potential dependence of the average length of the mono atomic wire was fully reversible.

The dependence of the average wire length on the electrochemical potential may reflect changes in the structure at the electrolyte / gold mono atomic wire interface. Sulfate anions adsorb on the surface of gold electrode at more positive than $0 \mathrm{~V}$. As the potential becomes positive, the coverage of sulfate increases gradually. ${ }^{8}$ Gradual increment in the plateau length at the positive polarization agrees well with the increment in the amount of the adsorbed sulfate anions on the surface. The result suggests that adsorbed sulfate ions contribute to stabilize mono atomic wire of gold in solution. At

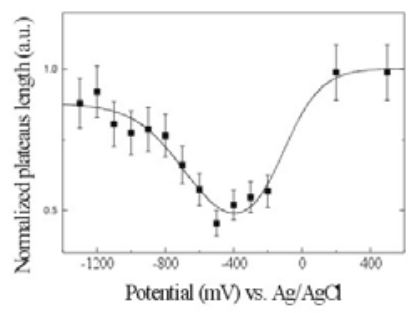

Figure 3. Length of $1 G_{0}$ plateau as a function of electrochemical potential in $0.1 \mathrm{M} \mathrm{Na}_{2} \mathrm{SO}_{4}$.

the negative polarization than $-0.4 \mathrm{~V}$, increment in the length of the plateau was also observed. Recently, we proved that certain atomic arrangement of $\mathrm{Ni}$ mono atomic wire was stabilized under the hydrogen evolution reaction. ${ }^{7}$ Contribution of evolved hydrogen molecule to stabilize the structure of the wire was considered in the system. Similar effect of hydrogen may also contribute to improve the stability of mono atomic wire of gold in the present system. Improved stabilities of the gold mono atomic wire both at the positive and the negative potential regions seem to reflect the amounts of adsorbed species onto the wire in solution. The Potential showing shortest stretched length $(-0.4 \mathrm{~V})$ is possible to reflect the potential of zero charge (pzc) of the present system. The pzc of gold electrode significantly depends on atomic arrangement and electrolyte at the interface. Different values ranging from $0.05 \mathrm{~V}$ to $0.3 \mathrm{~V}$ were shown in various single- and polycrystalline gold electrodes. ${ }^{9}$ The origin of the difference in the previously documented values is not clear at the present stage. Further detailed characterization on the structure of the gold mono atomic wire stretched in solution may clarify the origin.

Fully reversible change in the wire length of the gold mono atomic wire depending upon the electrochemical potential was achieved in the present system. The result proves the fact that the stability of the gold mono atomic wire can be controlled by the electrochemical potential. Further optimization of adsorbates and electrochemical potential are under way to find appropriate conditions to extend the length and the stability of the gold mono atomic wire in solution at room temperature.

In conclusion, the electric conductance of the gold nanoconstrictions was investigated in $0.1 \mathrm{M} \mathrm{Na}_{2} \mathrm{SO}_{4}$ solution under electrochemical potential control. A gold mono atomic wire was fabricated in solution at room temperature, in which thermal fluctuation of environment is rather large. Both at negative and positive polarization than $-0.4 \mathrm{~V}$, the length of the mono atomic wire increased significantly, possibly due to the increment of the amount of adsorbates to stabilize the gold mono atomic wire in solution. The reversible change in the length of the mono atomic wire proves the fact that the stability of the wire could be controlled by electrochemical potential at room temperature.

\section{References}

$1 \quad$ N. Agrait, A. L. Yeyati, J. M. van Ruitenbeek, Phys. Rep., 377, 81 (2003).

2 C. Cuevas, A. Levy Yeyati, A. Martín-Rodero, G. Rubio Bollinger, C. Untiedt, and N. Agraït, Phys. Rev. Lett., 81, 2990 (1998)

3 H. Ohnishi, Y. Kondo, and K. Takayanagi, Nature, 395, 780 (1998).

4 R. H. M. Smit, C. Untiedt, G. Rubio-Bollinger, R. C. Segers, and J. M. van Ruitenbeek, Phys. Rev. Lett., 91, 76805 (2003).

5 C. Shu, C.Z. Li, H. X. He, A. Bogozi, J. S. Bunch, and N. J. Tao, Phys. Rev. Lett., 84, 5196 (2000).

6 M. Kiguchi, T. Konishi, S. Miura, and K. Murakoshi, Trans. Mater. Res. Soc. Jpn., in press.

7 M. Kiguchi, T. Konishi, and K. Murakoshi, Appl. Phys. Lett., 87, 043104 (2005).

8 H. Uchida, M. Hiei, and M. Watanabe, J. Electroanal. Chem., 452, 97 (1998).

9 J. O’M. Bockris and S. U. M. Khan in "Surface Electrochemistry; A Molecular Level Approach”, Plenum Press, New York (1993). 
NOTE The diagram is acceptable in a colored form. Publication of the colored G.A. is free of charge.

For publication, electronic data of the colored G.A. should be submitted. Preferred data format is EPS, PS, CDX, PPT, and TIFF. If the data of your G.A. is "bit-mapped image" data (not "vector data"), note that its print-resolution should be 300 dpi.

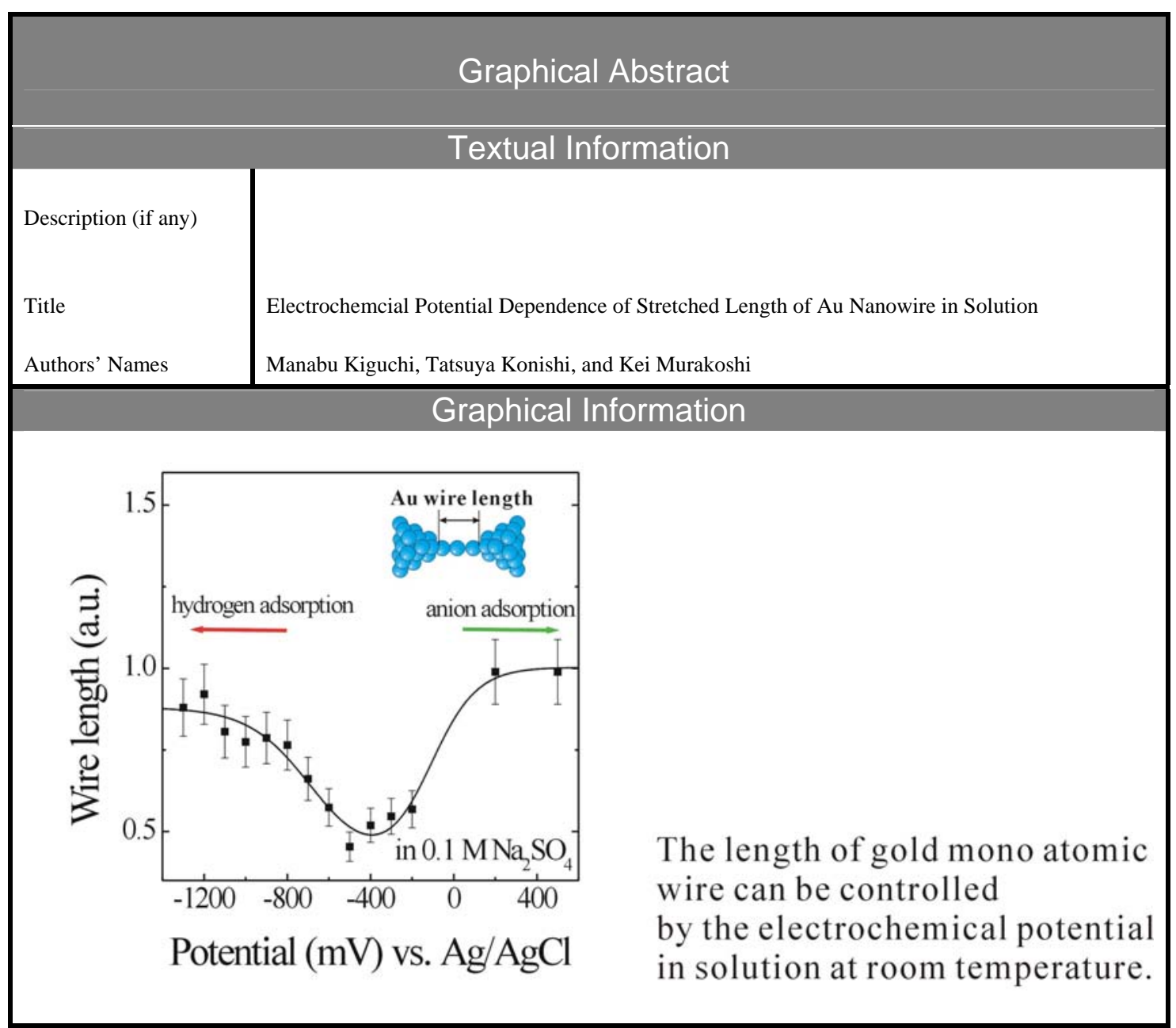

